2018-06

\title{
An exploration of peer-assisted learning in undergraduate nursing students in paediatric clinical settings: An ethnographic study
}

Carey, Matthew

http://hdl.handle.net/10026.1/11242

10.1016/j.nedt.2018.03.014

Nurse Education Today

Elsevier

All content in PEARL is protected by copyright law. Author manuscripts are made available in accordance with publisher policies. Please cite only the published version using the details provided on the item record or document. In the absence of an open licence (e.g. Creative Commons), permissions for further reuse of content should be sought from the publisher or author. 


\section{AN EXPLORATION OF PEER-ASSISTED LEARNING IN UNDERGRADUATE NURSING STUDENTS IN PAEDIATRIC CLINICAL SETTINGS: AN ETHNOGRAPHIC STUDY.}

Matthew C. Carey*, Anna Chick*, Bridie Kent*, Jos M. Latour*

*School of Nursing and Midwifery, University of Plymouth, Plymouth, UK

Word count: 4848

Corresponding author:

Matthew C. Carey

School of Nursing and Midwifery, Level 4, Rolle Building, Drake Circus, University of Plymouth, PL4 8AA.

matthew.carey@plymouth.ac.uk

(01752) 587508

@MattCarey16

Co-authors:

bridie.kent@plymouth.ac.uk

anna.chick@plymouth.ac.uk

jos.latour@plymouth.ac.uk

Acknowledgements:

This study was conducted as part of a larger PhD project funded by the School of Nursing and Midwifery, University of Plymouth. 


\section{Abstract:}

Background: Peer-assisted leaning relates to the acquisition of knowledge and skills through shared learning of matched equals. The concept has been explored within the field of nurse education across a range of learning environments, but its impact in practice is still relatively unknown. This paper reports on findings when observing paediatric undergraduate nursing students who engage in PAL within the clinical practice setting.

Objectives: The aim of this paper is to report the findings of a study undertaken to explore peer-assisted learning in undergraduate nursing students, studying children's health, in the clinical practice setting.

Design: A qualitative ethnographic study using non-participant observations.

Settings: A range of inpatient paediatric clinical settings across two teaching hospitals.

Participants: First, second and third year paediatric student nurses enrolled on a Bachelor of Nursing Programme.

Methods: Non-participant observations were used to observe a range of interactions between the participants when engaging in peer-assisted learning within the same clinical area. A total of 67 hours of raw data collected across all observations was analysed using framework analysis to draw together key themes.

Results: Of the 20 identified students across two hospitals, 17 agreed to take part in the study. Findings were aggregated into three key themes; 1 . Peers as facilitators to develop learning when engaging in peer-assisted learning, 2 . Working together to develop clinical practice and deliver care, 3. Positive support and interaction from peers to enhance networking and develop working structure.

Conclusions: Peer-assisted learning in undergraduate children's nursing students stimulates students in becoming engage in their learning experiences in clinical practice and enhance collaborative support within the working environment. The benefits of peer-assisted learning in current clinical practice settings can be challenging. Therefore, education and practice need to be aware of the benefits and their contribution towards future strategies and models of learning. 


\section{Introduction}

In recent years, more studies have begun to explore peer-centred learning methods to encourage student participation in education and promote critical thinking (Baeten et al, 2010). One such approach is peer-assisted learning (PAL), in which students acquire skills and knowledge through the active help provided by status equals or matched companions (Topping, 2005). The concept of PAL has been around for many years (Williams and Reddy, 2016); however, its advancement in nurse education has been affected by definition and lack of consistency (Secomb, 2008). There are many aspects of terminology that are associated with PAL, including "peer teaching" (Brannagan et al, 2013) "peer support" (Aston and Molassiotis, 2003) and "peer mentoring" (Li et al, 2010), which creates confusion.

Peer-assisted learning in the context of nurse education has been widely used within the simulated clinical skills environment, utilising junior and senior student nurses. Peer learning supported the acquisition of new clinical skills for junior students, whilst senior students consolidated teaching skills (Stables, 2012, Dumas et al, 2015). Increases in clinical knowledge and skills as well as clinical competence were seen by medical students within the context of clinical simulation (Seifert et al, 2016). Furthermore, PAL in undergraduate nursing education has resulted in enhancements in student's skills of communication, critical thinking and self-confidence particularly in theoretical settings (Christiansen and Jensen, 2008, Williams and Reddy, 2016).

Despite nursing students spending significant amounts of time learning in clinical settings, there appears to have been minimal exploration of PAL in the context of the clinical practice setting (Carey et al, 2016). Within the UK's Higher Education Institutes (HEls) 50\% of student learning takes place within the clinical practice environment, as outlined in standards for practice by the national Nursing and Midwifery Council (NMC, 2009). Currently, mentors who are registered nurses take on the role of supporting learning and assessing the competence of undergraduate nurses whilst in clinical practice (NMC, 2009, Casey and Clark, 2011). However, the perceptions of the quality of mentorship are variable as pressures on clinical workload limit the opportunities for students to work together with nurse mentors (Omansky, 2010, Kalischuk, 2013). At such times, undergraduate student nurses seek out each other for support (Carey et al, 2016). A report by the UK's Council of Deans of Health (2016) on educating the nurse of the future noted that students need to be equipped to teach others not just through mentorship, but informally in the clinical environment. They determined that this recommendation should be explored within undergraduate education and acknowledged PAL as an area for consideration (Council of Deans of Health, 2016). 
Therefore, with the gaps identified in the evidence-base for exploring PAL in practice, the challenges of current mentorship systems and the pressures facing learners and clinicians in clinical practice, there was a need to explore the contribution of PAL within the clinical setting on student learning.

\section{Objectives}

The aim of this paper is to report the findings of a study undertaken to explore peer-assisted learning (PAL) in undergraduate nursing students, studying children's health, in paediatric clinical settings.

The objectives were:

- To explore the extent of learning development across different year groups of child health nursing students when engaging in PAL.

- To identify if PAL provides opportunities for optimising education in clinical practice.

- To identify the types of interactions that occurred as part of PAL in the clinical setting.

\section{Methodology}

The philosophical underpinning of the study were taken from of an interpretivist research paradigm, in which reality needs to be interpreted as it is seen by the subjective experiences of individuals (Guba and Lincoln, 1994). Within this paradigm, a qualitative ethnographic approach was utilised in order to explore the impact of PAL on students learning within the clinical setting across two sites. One of the main benefits of an ethnographic approach is that it encourages the researcher to enter the environment of the participant to watch, listen and collect data where it is available (LoBiondo-Wood and Haber, 2014). Non-participant observations were identified as the most appropriate method for answering the research questions.

\subsection{Settings}

The study was conducted in two different teaching hospitals located within one region, in England. PAL has been implemented among nursing students since 2014. The process of PAL starts within the theoretical setting linked to clinical skills facilitation in the simulated 
environment. In the clinical settings registered nurse mentors of the students have been regularly updated about the PAL during monthly mentor updates with university lecturers.

\subsection{Ethics}

This study formed part of a larger project exploring PAL on enhancing the learning of undergraduate student nurses across multiple fields of nursing across different sites. Ethics for both sites of the study was granted by the university's students ethics committee and by the NHS. The study was conducted over two years between 2015 and 2016. Following ethical approval, students were given a participant information sheet outlining; the purpose of the study, its methods and how information would be kept confidential. Those agreeing to take part in the study met with the researcher within the practice environment to ask any further questions prior to obtaining written consent.

\subsection{Sample selection}

The students were purposefully selected from a range of first, second and third-year paediatric nursing students enrolled on a three-year undergraduate nursing degree programme, according their location and timing of placements across the two sites. Although the literature suggests PAL is provided amongst matched equals, in our study we defined matched equals as nursing students within the same programme and not by the same year of study. A sample size of 20 students were identified as having a placement across the two sites, with 17 agreeing to take part in the study. As the researcher was known to the participants, the students were approached by a third-party academic to avoid coercion and intimidation when seeking consent (Edens et al, 2011).

\subsection{Data collection}

Student participants were observed by the primary researcher over a period of two weeks at each site. Both sites included a range of paediatric inpatient environments according to student placement allocation. Interactions between groups of students from all levels of study were recorded using audio recordings and field notes between periods of 1-5 hours within the working shift patterns of student nurses. Before starting data collection, the ward staff and managers made in-patients and their families aware of the study and the observations. 


\subsection{Data analysis}

To promote anonymity, students were designated a code to represent their identity when collecting and referring to field notes. This was to ensure that no personal details were used as outlined within the participant information sheet. As a non-participant observer, the researcher was aware of the fact that they were known to the students. For this reason, the first hour of initial data recordings were not included within final analysis in consideration of the Hawthorne effect linked to participants observed (Sedgwick, 2012). It was noted that after this initial time students appeared to be less aware of the researcher and focused themselves upon engaging with their peers. Interestingly Monahan and Fisher (2010) argue that the performance of the participants, whether staged or influenced by the observer can often reveal profound truths about social and cultural phenomena.

Framework analysis was used to analyse the data, based upon the tool created by Ritchie and Spencer (1994). A defining feature of the framework methods through its step by step stages is to use a matrix output (rows and columns) of summarised data (codes) to provide a structure for the researcher to systematically reduce data for analysis by case and code (Ritchie et al, 2013). Normally associated with interviews, the principles for this method of data analysis can be utilised for other textual data such as field notes and recorded data from observations (Gale et al, 2013). A seven step process was utilised to analyse over 35 hours of raw data to draw together key themes (Figure 1). These steps were undertaken by the primary researcher, with a second coder utilised to ensure systematicity, clarity and transparency when analysing data (Hall et al, 2005). Visitation of key stages were discussed between the researcher and second coder to develop and compare themes. These findings were developed into a working framework model that could be utilised to analyse the data from site two and synthesised into a more trustworthy framework model. At site two, another 32 hours of observations were collected. Within the findings, the student commentary are coded. For example [S1,StA,StC,W1]: S1 represent hospital 1 and S2 is hospital 2 where the observations took place; StA is a first year student, StB a second year student, and StC a third-year student; W1 is adolescent ward, W2 is acute paediatric ward; W3 is paediatric high dependency ward; W4 is paediatric assessment unit, and W5 is paediatric oncology ward.

Figure 1: the framework process, adapted from Ritchie and Spencer (1994). 


\section{Results}

Data revealed three main themes contributing to impact of peer-assisted learning on enhancing the learning of undergraduate paediatric nurses. These themes were: peers as facilitators to develop learning when engaging in PAL, working together as peers to develop clinical practice and deliver care and finally, positive support and interaction from peers to enhance networking and develop working structure.

\subsection{Peers as facilitators to develop learning when engaging in PAL}

Theme one conveys the positive acknowledgement of peers acting as informal facilitators of learning towards their peer colleagues. These types of interactions occurred between student nurse peers across both sites when engaged in PAL Observations recorded examples of opportunities that had the potential to optimise education amongst peers. These were demonstrated through systems of informal teaching support to offer advice and guidance to other peer colleagues. In one observation a third-year student nurse offers some teaching demonstration related to medication administration, discussing the formula for drug calculation and provides this to a group of three first years: From my experience I find it easier to use the NHS [National Health Service-UK] example as it's easier to remember each part and relate this to the calculation. This is further supported by: I like the NHS method that some of our group use - what you Need, what you Have and what Solution. This one is easier to remember I think. [S1,StA,StC,W1]. It is noted the value that the firstyear student nurses gain through this demonstration: this is great, thanks I think we give this one a go next. [S1,StA,W1].

Peers across all level of study had an open policy of communication to ask questions and seek support to enhance their learning in the context of the clinical environment. Questioning enabled peer pairs to openly engage and confirm their understanding of various clinical tasks with their peer colleagues. In an observation a peer pair noted the importance of being able to seek assurance and guidance with their peer colleagues: It can be really stressful sometimes, especially when it's busy. It helps to have someone else on hand to ask questions and if I need a bit of help with ward tasks [S1,StA,W2]. This was further observed within most peer pairing who seemed to have limited restriction on the types of questions that were asked in relation to clinical practice.

Peers across both sites and within different years of study offered facilitation when discussing clinical competencies and how these related to clinical practice. As part of student development peer pairs were observed to plan and relate clinical and academic 
assessment assigned by the university, examples including; clinical examinations, creating development plans for practice and the implementation of clinical competencies set by the university. In an observation, two students take time out in the day for completing their development plans. They discuss what competencies they believe relates to practice and how they can relate this to their current placement: 'your domain for communication fits with all placements, but only certain skills can be completed in the acute areas. Like clinical observations, we could only do these here.' [S2,StC(x2),W3].

\subsection{Working together to develop clinical practice and deliver care}

Theme two considers the active and positive relationship between the student nurses across both sites as they engage in peer-assisted learning. The context of learning provides examples of where peer interactions shape the development of the students' own clinical practice. This was seen through examples of discussion, which related to determining best practice standards, but then applying this to the care of the patient. In one example, second year students working together as a peer pair challenge each other's knowledge regarding the expectations of giving antipyretics to children and why they shouldn't be giving them to every child. They discuss the role of the student nurse in providing the parent with advice and guidance to ensure effective health promotion. Student's give practical examples of applying these situations to practice but relate these back to national guidelines to reinforce standards of care: Guidelines, say only when it is high beyond 39 degrees $C$, you need the temperature there to fight infection, but all parents want their child to have Calpol without knowing why it's not needed. Yeh, you have to find out the reason why they need it before making the right decision to give it [S2, $\mathrm{StB}(\mathrm{x} 2), \mathrm{W} 4]$.

Students demonstrated learning and development, through positive PAL interaction, within clinical practice to plan and develop care for patients. Within one observation, a student pair take time to discuss the patient under their supervision to determine what needs to be prioritised and to be carried out as part of their ongoing care before instigating this. Within their pairings, students would often share in the learning experience of implementing and providing care for patients when engaging in PAL. In frequent observations across sites and different parings, students would undertake routine observations of patients in the bays and side cubicles. Prior to undertaking these tasks students would establish the role they would like to perform. Students were also accommodating to share out these roles to ensure that experiences were shared in turns: I'm happy if you want to complete the obs and I'll write 
them down, but we can always swap later with the next one? [S1,StA,StC,W1]. This approach was widely accepted by multiple pairs of students.

Between periods of implementing care, student nurses were observed and seen to share and relate their experiences of clinical practice to aid their understanding and develop their learning. In one example a third-year student working with a junior first year provides a direct identification of experience of when they had to take observations on a challenging patient: the infant was distressed, which would raise their B.P. (blood pressure), so this wouldn't offer the best result to record [S1,StA,StC,W5]. This example led into a discussion about how these experiences can be shared to help learn from them and tips on how to develop your own practice as a student nurse. The senior student shares experiences of when it is not appropriate to approach a patient for observation if they are stressed and how you can encourage them in other ways: You could distract them with a toy or something, then you can attempt to put the cuff on them when they are settled [S1,StA,StC,W5].

\subsection{Positive support and interaction from peers to enhance networking and develop working structure}

The focus of theme three centred on demonstrating the networking elements arising from peer interaction within the clinical environment. Peer support was seen as a vital element for students across participants at different levels of study and interaction. Students when engaging in PAL often used each other for navigating the clinical environment especially in the early stages of a new placement. In one observation a paring of two first year students discuss some of the challenges of finding their way around placement, especially when it is new. One student makes a point to note how they find it beneficial to have another student colleague around when working in a stressful clinical area: It can be really stressful sometimes, especially when it's busy. It helps to have someone else on hand to ask some simple questions and if I need a bit of help with ward tasks [S1,StA,W2]. This is a commonality, which was reciprocated by the other peer within the pair: Yeah, you are right, I feel the same, it's nice to see a friendly face [S1,StA,W2].

The engagement of PAL by both junior and senior peers highlighted a culture whereby they would feel confident to openly discuss the structure of the clinical area and consider where they fit into this environment as student nurses. In early observations, students were seen to regularly discuss their approach towards the day-to-day tasks and the structure of working within the clinical area. Students, especially within pairings of first years were interested in the process of where they fit into this structure and how they adapt to these new placement 
experiences. In one observation, a peer pair who are new to the placement use the start of the day to consider the task ahead: we always check the drugs charts first, then go see the patients. We can do this as students. Let's do that first then we can go through what we need to do next [S1,StA,W2].

Peers seemed to work well together in their pairings when engaging in PAL. The acceptance towards being part of a pair was seen through observing different groups and years of study across different sites. Students were open to the opinions of their peer colleagues when engaging in shared learning and networking, which in one observation appeared to be underpinned by mutual respect seen between two peers. This was demonstrated in the way that they stood alongside each other by the desk, engaged in a patients assessment paperwork and discussed/confirmed what it is that they are have just completed. Both peers shared thoughts and interpretation of the information, giving each other opportunity to speak and share in their ideas before agreeing on the outcome: The mum mentioned that they hadn't been feeding too well, do you agree with this? Yep, I see that you've recorded it here, but they are having wet nappies, which is good. Yeah, I agree [S2,StB,W4].

Networking also extended to opportunities for social interactions in which students could offload stress and seek emotional support from their peer colleagues. In one observation: students during a period of respite from clinical task entered into a conversation which enabled them to reflect on the benefits of working together and how they are able to use each other for emotional support: 'it's good to have someone to chat with and have a moan, especially when you have a busy day. I know you are there if I need you and that takes some of the pressure off! [S1,StA,W2]

\section{Discussion}

The findings presented in this paper capture benefits arising from engaging in peer-assisted learning within clinical practice. These include peers being informal facilitators to develop learning, as well as the provision of advice and guidance. This finding is supported by that of Walsh, who explored the effects of social interaction of mental health student nurses when in community placements (Walsh, 2015). Walsh also noted ad hoc situations of informal facilitation of learning whereby some students would provide teaching and show other peer colleagues how to do things when on placement.

Opportunities for optimising education in clinical practice appeared limited within the data, however, support was provided by peers through shared learning experiences. These 
opportunities were extended by peers from all level of study related to a variety of learning situations, but especially when providing patient care. In a systematic review, Davis et al (2016) explored the influence of workplace culture on qualified nurses' learning experiences. They noted the importance of peer support in shared learning experiences to facilitate and encourage other peers to develop in their learning. They also reported that qualified nurses who did not have access to support from peers during unfamiliar tasks were likely to have limited learning outcomes compared to having the opportunity to participate in new activities supported by peers (Davis et al, 2016).

Using peers to support student learning in practice is a philosophy shared by a coaching strategy for undergraduate nursing students known as the Collaborative Learning in Practice (CLiP) model (Lobo et al, 2014). This model, developed in Amsterdam, is one in which student-led and peer learning is used to encourage students in their learning in clinical settings. Coaches, rather than mentors, used less telling and teaching, and more questioning, enquiry and prompting to encourage students to plan and assume responsibility for the care they provide (Lobo et al, 2014). Such behaviours were observed in this study and captured within theme two, whereby students of all levels of study had an open policy of communication and questioning and seek support to enhance their learning in the context of the clinical environment.

The opportunities for optimizing education, despite its limitation within the data, did note frequency in the support of peers to discuss and support each other in planning assessment competencies. This is a finding that is sparsely reported within the current nursing literature. One study related to PAL with medical students in the clinical setting supported the importance of PAL for exam focused learning among students (Bennett et al, 2015). Interestingly, multiple participants across the two site put large emphasis on bring up these topics as points of discussion and to utilize their peers in planning and negotiating appropriate clinical competencies for the placement environment. Despite limited links to the aims of the study, the acknowledgement of general discussion amongst peers related to planning competencies offers an area for further research, to indicate whether this support has influence on student education when implementing these in clinical practice.

The types of interactions between peers were varied and ad hoc. In an example from the data a senior student nurse used informal situations to teach their junior peer colleagues. Furthermore, junior student nurses offering their support across different levels of study, but particularly noted positive support from peers that then help navigate practice, especially in times of stress. This was also identified by Walsh (2015). When faced with stressful 
situations students found the emotional support of other peers valuable to talk to someone who understands (Walsh, 2015). The notion of peer support to develop some structure within the work environment appeared to be important early on within a new placement for some of the peers. This was also identified by Roberts (2008) who explored the importance of peers for learning in practice. For most of the students, they reported the company of peers to be important within a new placement, sticking together and being able to swap experiences and anecdotes.

\subsection{Limitations of the study}

One limitation of the study focuses on the timing of the observations conducted. Following ethical approval, periods of observation were limited to when the students were allocated on placement. Therefore, students were purposely selected based upon the allocation and timing of these. This added some restriction to the numbers in Child Health nursing students due to the size and allocation of the placement environments. Another limitation is the restriction between the years of study of participants available for observation. In our study we defined matched equals as being a student nurse, regardless of the stage of study. Ideally the observations should have been conducted between students at the same stage of study. We also acknowledge the 65 hours of transcription as limited observation time for an ethnographic study. Finally, the study included mainly female participants with only one male participant. Further research is needed to identify gender differences when engaging in PAL.

\section{Conclusion and recommendations}

The benefits of peer-assisted learning are an important consideration for the future of both nurse education, also to consider that students have much to gain from one another when engaging in PAL in the clinical practice setting. It is clear that the learning, which students experience in the real world setting have many varied and complex challenges that they must face. Therefore, in light of recommendations for educating the nurse of the future Higher Education Institutes and mentors/educators need to be aware of the benefits of PAL and how it contributes towards future strategies and models of learning. As this was a small qualitative study, future recommendations include the need to explore other fields of nursing to determine similarities and further influences of PAL amongst nursing students.

\section{Conflicts of interest}

The authors declare no conflict of interest. 


\section{Acknowledgements}

This study was conducted as part of a larger PhD project funded by the School of Nursing and Midwifery, University of Plymouth. 


\section{References:}

Aston, L., Molassiotis, A. 2003. Supervising and supporting student nurses in clinical placements: the peer support initiative. Nurse Educ. Today. 23(3), 202-210.

Baeten, M., Struyven, K., Dochy, F. 2010. Using student-centred learning environments to stimulate deep approaches to learning: Factors encouraging or discouraging their effectiveness. Educational Research Review. 5(3), 243-260.

Bennett, D., O'Flynn, S., Kelly, M. 2015. Peer assisted learning in the clinical setting: an activity systems analysis. 20(3), 595-610.

Brannagan, K., Dellinger, A., Thomas, J., Mitchell, D., Lewis-Trabeaux, S., Dupre, S. 2013. Impact of peer teaching on nursing students: perceptions of learning environment, selfefficacy, and knowledge. Nurse Educ. Today. 33 (11), 1440-7.

Carey, M.C., Kent, B., Latour, J.M. 2016. The role of peer-assisted learning in enhancing the learning of undergraduate nursing students in clinical practice: a qualitative systematic review protocol. JBI Database of System Reviews and Implementation Reports. 14 (7), 117123.

Casey, D.C., Clark, L. 2011. Roles and responsibilities of the student nurse mentor: an update. British Journal of Nursing. 20 (15), 933-937.

Christiansen, B., Jensen, K. 2008. Emotional Learning within the framework of nursing education. Nurse Education in Practice. 8 (5), 328-334.

Council of Deans of Health Shape of Caring Advisory Group. 2016. Educating the Future Nurse - a paper for discussion. Council of Deans of Health.

Daley, L.K., Menke, E., Kirkpatrick, B., Sheets, D. 2008. Partners in practice: a win-win model for clinical education. Journal of Nursing Education. 47 (1), 30-32.

Davis, K., White, S., Stephenson, M. 2016. The influence of workplace culture on nurses' learning experiences: a systematic review of qualitative evidence. JBI Database of Systematic Reviews and Implementation Reports. 14 (6), 274-346.

Dumas, B.P., Hollerbach, A.D., Stuart, G.W., Duffy, N.D. 2015. Expanding Simulation Capacity: Senior-Level Students as Teachers. Journal of Nurse Education. 54 (9), 516-519.

Edens, J.F., Epstein, M., Stiles, P.G., Poythress, N.G. 2011. Voluntary Consent in Correctional settings: Do Offenders Feel Coerced to Participate in Research? Behavioural Science and the Law. 29 (6), 771-795.

Gail, N.K., Heath, G., Cameron, E., Rashid, S., Redwood, S. 2013. Using the framework method for the analysis of qualitative data in multi-disciplinary health research. BMC Medical Research Methodology. 13 (1), 1-8.

Guba, E. G., Lincoln, Y. S. 1994. Competing paradigms in qualitative research. In N. K. Denzin \& Y. S. Lincoln (Eds.), Handbook of qualitative research (pp. 105-117). London: Sage.

Hall, W.A., Long, B., Bermbach, N., Jordan, S., Patterson, K., 2005. Qualitative Teamwork Issues and Strategies: Coordination Through Mutual Adjustment. Qualitative Health Research. 15 (3), 394-410. 
Kalischuk, R.G., Vandenberg, H., Awosogga, O. 2013. Nursing preceptors speak out: an empirical study. Journal of Professional Nursing. 29 (1), 30-38.

Li, H.C., Wang, L.S., Lin, Y.H., Lee, I. 2010. The effect of a peer-mentoring strategy on student nurse stress reduction in clinical practice. International Nursing Review. 58 (2), 203210.

LoBiondo-Wood, G., Haber, J. 2014. Nursing Research: Methods and Critical Appraisal for Evidence-Based Practice. $8^{\text {th }}$ Edition. Elsevier.

Lobo, C., Arthur, A., Laittimer, V. 2014. Collaborative Learning in Practice (CLiP) for preregistration nursing students. University of East Anglia.

Monahan, T., Fisher, J.A. 2010. Benefits of "Observer Effects": Lessons from the Field. Qualitative Research. 10 (3), 357-376.

Nursing and Midwifery Council. 2009. Standards to support learning and assessment in practice. [internet]. [cited 2017 September 4]. Available from: http://www.nmcuk.org/Documents/Standards/nmcStandardsToSupportLearningAndAssessmentInPractice.pdf

Osmansky, G.L. 2010. Staff nurses' experiences as preceptors and mentors: an integrative review. Journal of Nursing Management. 18 (6), 697-703.

Ritchie, J., Spencer, L. 1994. Qualitative data analysis for applied policy research. In: Bryman, A., Burgess, R.D. (Eds). Analyzing qualitative data. London, Routledge. pp.173194.

Ritchie, J., Lewis, J., McNaughton-Nicholls, C., Ormston, R. 2013. Qualitative research practice. A guide for social science students and researchers. Sage. London.

Roberts, D. 2008. Learning in clinical practice: the importance of peers. Nursing Standard. 23 (12), 35-41.

Secomb, A. 2008. A systematic review of peer teaching and learning in clinical education. J Clin Nurs. 17 (6), 703-716.

Sedgwick, P. 2012. The Hawthorne effect. BMJ. 344, pp:1-2.

Seifert, L.B., Schaack, D., Jennewein, L., Steffen, B., Schulze, J., Gerlach, F., Sader, R. 2016. Peer-assisted Learning in a student-run free clinic project increases clinical competence. Medical Teacher. 38 (5), 515-522.

Stables, I. 2012. DEVELOPMENT OF CLINICAL SKILLS: THE CONTRIBUTION OF PEER LEARNING. Learning Disability Practice. 15 (8), 12-17.

Topping, K.J. 2005. Trends in peer learning. Educational Psychology. 25 (6), 631-45.

Walsh, A. 2015. The effect of social interaction on mental health nurse student learning. Nurse Education in Practice. 15 (1), 7-12.

Williams, B., Reddy, P. 2016. Does peer-assisted learning improve academic performance? A scoping review. Nurse Education Today. 42, 23-29. 


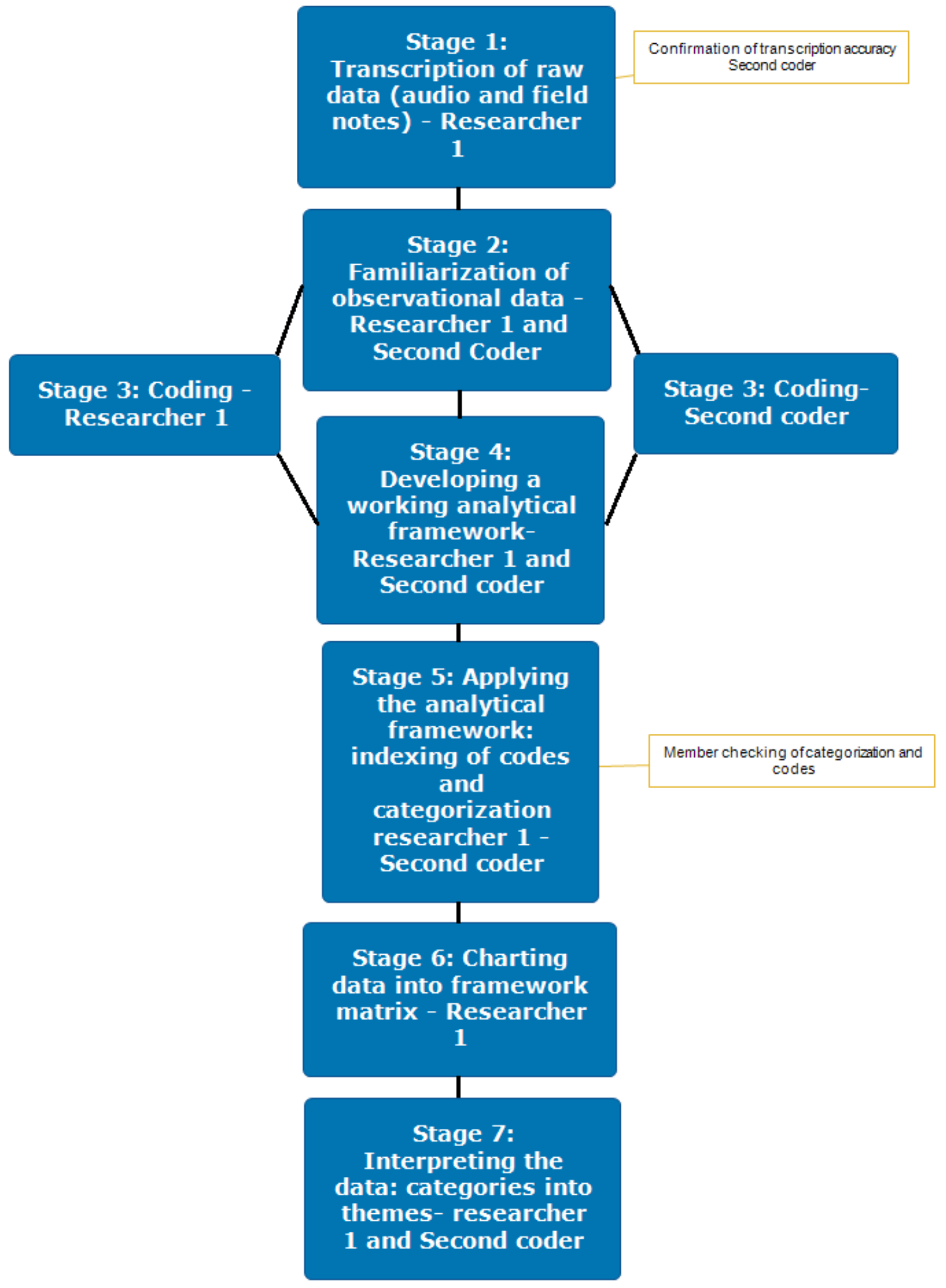

